\title{
Asbestos and malignant mesothelioma: foreword
}

\author{
Reviews of a symposium entitled "Malignant Mesothelioma" organized \\ by the Study Group on Fibrous and Particulate Substances of the 77th Annual Meeting \\ of the Japanese Society for Hygiene, 2007
}

\section{Takemi Otsuki}

Published online: 28 February 2008

(c) The Japanese Society for Hygiene 2008

Among the symposia organized by various study groups in the Japanese Society for Hygiene (JSH), the Study Group on Fibrous and Particulate Substances (SGFPS) presented a symposium entitled "Malignant Mesothelioma," chaired by Prof. Takemi Otsuki (Kawasaki Medical School, Japan) on 28 March 2007, as a part of the program of the 77th Annual Meeting.

It is common knowledge that asbestos exposure causes asbestos-related diseases such as asbestosis, lung cancer and malignant mesothelioma, not only in people who have handled asbestos in the work environment, but also in residents who live near factories that handle asbestos. It has been a big medical and social problem since the summer of 2005.

At the 76th Annual Meeting of the JSH, held in 2006 at Ube, Yamaguchi, Japan, the SGFPS held a symposium entitled "Asbestos: Science and Society," chaired by Prof. Eiji Yano (Teikyo Univeristy, Japan) and Prof. Otsuki. In that symposium, Dr. Kenji Morinaga (National Institute of Industrial Health, Japan) talked about "past and present asbestos issues in Japan." Professor Shuichi Adachi (Sagami Women's University, Japan) focused on "risk analysis of asbestos exposure from ambient air." In addition, "disposal of asbestos-containing waste" was presented by Dr. Ayako Takata (St. Marianna University School of Medicine, Japan) and "an explanation of fibrous material experimental methods" was given by Dr. Yuichiro Kudo (Kitasato University School of Medicine). From these presentations, the SGFPS hoped that the audience and

T. Otsuki $(\bowtie)$

Department of Hygiene, Kawasaki Medical School,

Kurashiki 701-0192, Japan

e-mail: takemi@med.kawasaki-m.ac.jp members of JSH could clarify and update their knowledge of asbestos.

Thereafter, a new law to economically contribute to people who are suffering from or have been suffering from and have died of asbestos-related diseases, particularly malignant mesothelioma and lung cancers, was passed in March 2007. In addition, the number of fatalities due to malignant mesothelioma has been increasing rapidly, because approximately 40 years have passed since the huge increase in the amount of asbestos used in Japan since the 1960s and 1970s. Furthermore, it is assumed that the number of mesothelioma cases will increase much more until 2020 or later, according to the amount of asbestos used in Japan. For these reasons, it is very important to determine the pathophysiology of malignant mesothelioma from the viewpoints of clinical and preventive medicine.

At the symposium held in 2007, the SGFPS provided an opportunity to listen to reviews related to malignant mesothelioma from specialists who are associate members of the JSH in order to improve recognition of this disease and to create preventive measures for this disease in the field of hygiene.

Professor Kouki Inai (Hiroshima University, Japan) presented the "pathology of mesothelioma" and Dr. Yoshitaka Sekido (Aichi Cancer Center Research Institute, Japan) focused on the "molecular biology of mesothelioma." In addition, Prof. Okio Hino (Juntendo University School of Medicine, Japan) lectured on a "tumor marker for asbestos-related mesothelioma (asbestoma)." Finally, Prof. Takashi Nakano (Hyogo College of Medicine, Japan) presented "malignant mesothelioma: diagnosis and treatment strategies."

In this special issue of Environmental Health and Preventive Medicine, all of the contributors to the symposium 
review their specialities. Tremendous efforts should be applied to cure patients with malignant mesothelioma and to prevent the occurrence of the disease. This special issue of EHPM may provide ideas and suggestions for all members of the JSH regarding how we should proceed to fight this disastrous health disorder. 